\title{
Adaptive Cluster Sampling Based on Ranked Sets
}

\author{
Girish Chandra $^{1}$, Neeraj Tiwari ${ }^{2}$, and Hukum Chandra ${ }^{3}$
}

\begin{abstract}
In many surveys, characteristic of interest is sparsely distributed but highly aggregated; in such situations the adaptive cluster sampling is very useful. Examples of such populations can be found in fisheries, mineral investigations (unevenly distributed ore concentrations), animal and plant populations (rare and endangered species), pollution concentrations and hot spot investigations, and epidemiology of rare diseases. Ranked Set Sampling (RSS) is another useful technique for improving the estimates of mean and variance when the sampling units in a study can be more easily ranked than measured. Under equal and unequal allocation, RSS is found to be more precise than simple random sampling, as it contains information about each order statistics. This paper deal with the problem in which the value of the characteristic under study on the sampled places is low or negligible but the neighbourhoods of these places may have a few scattered pockets of the same. We proposed an adaptive cluster sampling theory based on ranked sets. Different estimators of the population mean are considered and the proposed design is demonstrated with the help of one simple example of small populations. The proposed procedure appears to perform better than the existing procedures of adaptive cluster sampling.
\end{abstract}

\section{Introduction}

Thompson (1990) introduced the Adaptive cluster sampling as an efficient sampling procedure for estimating totals and means of rare and clustered populations. In adaptive cluster sampling, an initial sample of units is selected by some ordinary sampling scheme, and, whenever the variable of interest of a unit in

\footnotetext{
1 Division of GC(R), Tropical Forest Research Institute, Mandla Road, Jabalpur-482021, India; gchandra23@yahoo.com.

2 Department of Statistics, Kumaun University, SSJ Campus Almora, Uttarakhand-263601, India; kumarn_amo@yahoo.com.

3 Division of Sample Survey, Indian Agricultural Statistics Research Institute, New Delhi110012, India; hchandra12@gmail.com.
} 
the sample satisfies a previously specified condition ' $\mathrm{C}$ ', neighbouring units are added to the sample. If any of the newly added units satisfy ' $\mathrm{C}$ ', units in their neighbourhoods are also added until the sample includes all the neighbours of any unit satisfying the condition ' $C$ '. As noted by Thompson (1990), an adaptive cluster sampling scheme can be used to investigate a rare contagious disease. First of all, a simple random sample of people are selected and tested for the disease. If a person tests positively, then all the friends and contacts of that person are also tested. If one of the contacts tests positively, then all that person's contacts are tested, and so on. Roesch (1993) used the design for a survey of forest trees. Thompson and Seber (1996) described some examples of rare species, rare diseases and environmental pollution studies where the use of adaptive sampling scheme can be highly beneficial. The condition for extra sampling might be the presence of the rare animal or plant species, high abundance of a spatially clustered species, detection of "hot spots" in an environmental pollution study, high concentration of mineral ore or fossil fuel, observation of a rare characteristic of interest in a household survey, and so on. For more details on adaptive cluster sampling, one may refer to Thompson (1991a, 1991b), Chaudhuri et al. (2004), Salehi and Seber (2004), Thompson and Seber (1996), Blanke (2006) and Hu and Su (2007).

The procedure for selecting initial sample is most important to increase the precision of the estimates of mean and variance. While most of the researchers have used the method of simple random sampling to select the initial sample, we investigated the possibility of using Ranked Set Sampling (RSS) in selecting the initial sample. RSS, introduced by McIntyre (1952), is a sampling scheme that can be utilized to potentially increase precision and reduce costs when actual measurement of the variable of interest is costly or time-consuming but the ranking of the set of items according to the variable can be done without actual measurements. Such situations normally arise in environmental monitoring and assessment that require observational data. For example, the assessment of the status of hazard waste sites is usually costly. But, often, a great deal of knowledge about hazard waste sites can be obtained from records, photos and certain physical characteristics that can be used to rank the hazard waste sites. In certain cases, the contamination levels of hazardous waste sites can be indicated either by visual inspection such as defoliation or soil discoloration, or by inexpensive indicators such as special chemically-responsive papers, or electromagnetic reading. RSS is a two-phase sampling design that identifies sets of field locations, utilizes inexpensive measurements to rank locations within each set, and selects one location from each set for sampling.

In the simplest form of RSS or RSS with equal allocation, first a simple random sample of size $\mathrm{k}$ is drawn from the population and the $\mathrm{k}$ sampling units are ranked with respect to the variable of interest, say $X$, without actual measurements. Then the unit with rank 1 is identified and taken for the actual measurement of $X$. The remaining units of the sample are discarded. Next, another 
simple random sample of size $\mathrm{k}$ is drawn and the units of the sample are ranked by judgment, the unit with rank 2 is now taken for the measurement of $\mathrm{X}$ and the remaining units and discarded. This process is continued until a simple random sample of size $\mathrm{k}$ is taken and ranked and the unit with rank $\mathrm{k}$ is taken for measurement of $X$. This whole process is referred to as a cycle. This cycle is then repeated $\mathrm{m}$ times which yields a ranked set sample of size $n=m k$. In this procedure each order statistics is repeated same number of times i.e. $\mathrm{m}$. If this number is not same for some or all order statistics the procedure is referred as RSS with unequal allocation. The relative precision (RP) of RSS compared with SRS is always an increasing function of set size (k). Use of appropriate allocation model for all order statistics further increases the gain in RSS.

RSS has been satisfactorily used to estimate pasture yield by McIntyre (1952, 1978), forage yields by Halls and Dell (1966), mass herbage in a paddock by Cobby et al. (1985), shrub phytomass by Martin et al. (1980) and Muttlak and McDonald (1992), tree volume in a forest by Stokes and Sager (1988), root weight of Arabidopsis thaliana by Barnett and Moore (1997) and bone mineral density in a human population by Nahhas, Wolfe, and Chen (2002). Patil, Sinha, and Taillie (1994) discussed some other situations where RSS may be applied. A complete review of the applications and theoretical work on RSS can be found in Kaur et al. (1995) and Chen, Bai, and Sinha (2004).

When carrying out environmental pollution studies, the following situation may commonly encounter. In most of the sampled places, the pollution is low or negligible. However, the neighbourhoods of these places may have a few scattered pockets of high pollution. Under such situations, Thompson (1996) proposed an adaptive design based on order statistics in which an initial simple random sample of pollution readings on $\mathrm{n}$ sites was taken, yielding the ordered readings $y_{(1)}<y_{(2)}<\ldots \ldots . .<y_{(n)}$. This design is helpful to choose the criterion $\mathrm{C}$ but there is a good chance that most of the pockets of high pollution are missed. To overcome this difficulty, there arises a need of an adaptive scheme in which each order statistics are considered. This is achieved with the help of the proposed design in which we use the technique of ranked set sampling to select the initial sample.

To demonstrate the applicability of the proposed procedure, we consider a real life situation. In determining the estimate of density and distribution of rare or endangered plant species, generally the information about the abundance is not available to us. But these types of species are found in the form of clusters. Also there is a large variation in the areas of clusters and there may be a good chance that neighbourhoods of small clusters may have clusters with larger areas. Under such circumstance the strategy to use SRS or other sampling procedures in first phase is not appropriate. Because we may omit such clusters while using these designs, which may have come in the final sample using RSS. The reverse situation may also exist i.e. the neighbourhood of larger area clusters may have very small clusters. When we use RSS, the probability of omitting such clusters 
becomes more than the other procedures. Using RSS in the first phase of the design, all type of clusters from smallest to largest are considered and due to the variations in the neighbourhood of the clusters in the proposed design, we are in a position to consider high abundance of rare plant species with minimum cost and time.

In the present paper, we propose an adaptive cluster sampling theory based on ranked sets. In this theory the initial sample is selected by the method of ranked set sampling and if the measured values of the units in initial sample satisfies the pre-specified condition $\mathrm{C}$ then their neighbourhoods are added as well. The proposed theory appears to be highly appropriate for the environmental situations discussed in the penultimate paragraph. Since the relative precision of RSS compared with SRS is always an increasing function of set size (k), the proposed procedure yields higher precision as compared to existing procedures as $\mathrm{k}$ increases.

Details of the proposed design with the notations used are given in the Section 2. Section 3 describes the estimators of the population mean. In Section 3.1, the estimator based on only initial sample, has been considered. Section 3.2 deals with an estimator based on initial intersection probabilities along the lines of Thompson (1990). Improvement of the estimators using the Rao-Blackwell theorem has been attempted in Section 3.3. In Section 4, the proposed design is demonstrated with the help of a simple example taken from a small artificial population. Section 5 concludes the findings of the present paper.

\section{The proposed sampling design}

Suppose that we have a finite population of $\mathrm{N}$ units with labels $1,2 \ldots \mathrm{N}$ and with associated variables of interest $\mathbf{y}=\left(y_{1}, y_{2}, \ldots \ldots \ldots y_{N}\right)$. Our interest is to estimate the population mean of the $\mathrm{y}$-values, given by $\mu=\frac{1}{N} \sum_{i=1}^{N} y_{i}$. To define the neighbourhood of each unit $i$, we say that if $i$ is a neighbourhood of the unit $\mathrm{j}$ then unit $\mathrm{j}$ is also the neighbourhood of unit $i$. A typical neighbourhood might be the unit itself together with the four units with common boundaries, when the whole population is arranged in a systematic grid pattern. Thus a neighbourhood of unit $i$ consists of five units in a cross shape shown in Figure 1. The neighbourhoods do not depend on the y-values of population. The unit $i$ is said to satisfy the condition of interest $C$ if the associated $y$-value $\left(y_{i}\right)$ is in a specified set C. 


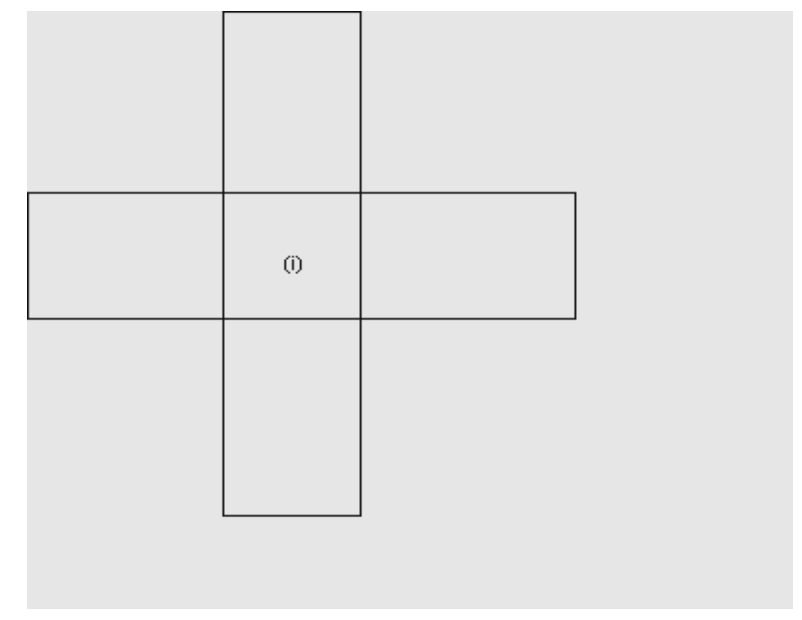

Figure 1: Neighbourhood of unit i for grid pattern population.

The proposed design, for selecting the final sample, can be classified into two phases as follows:

The first phase of an adaptive cluster sampling design based on ranked sets consists of selecting a ranked set sample of size $\mathrm{n}$ from the population of $\mathrm{N}$ units. Throughout this paper, we have taken $m=1$ without any loss of generality.

In the second phase of the proposed design we add neighbourhoods adaptively of the measured units in the first phase if the units satisfy the pre-specified condition of interest $C$. If any of these added units satisfies $C$ then there neighbourhoods are also added and so on until we end up with a cluster that has a boundary of units that do not satisfy $\mathrm{C}$. These boundary units of each cluster are called edge units. The final sample then consists of $n$ (not necessarily distinct) clusters, one for each unit selected in the initial sample.

An example is illustrated in Figure 2, in which the aim is to estimate the concentration of the contamination level of the pollution over a specified site. The population (site) is divided into $10 \times 10$ square plots; each plot is a unit of the population. The y-value of unit $i$ represents the contamination level and is demonstrated in each cell in Figure 2. A unit satisfies the condition of interest $\mathrm{C}$ if it contains contamination level greater than or equal to 1 , i.e. $y_{i} \geq 1$. Three random sets of units each of size 3 are drawn without replacement from the population and ranked according to the y-value. The units of the first, second and third sets are shown in Figure 2 by 'bold', 'underlined' and 'bold and underlined', respectively. The units taken for measurement in the initial sample is the first smallest, the second smallest and the largest from the first, second and third sets respectively. This initial sample of units is shown in the Figure 3 by 'red bold' outline. Applying the second phase of the design gives the final sample, which is shown in Figure 3 by 'bold' and 'red bold' outline. 


\begin{tabular}{|c|c|c|c|c|c|c|c|c|c|}
\hline 0.32 & 0.8 & $\mathbf{1 . 1}$ & 2.3 & 5 & 0.46 & 0.13 & 0.59 & 0.32 & 0.35 \\
\hline 0.56 & 0.6 & 5 & 2.98 & 4.9 & 0.35 & 0.14 & 0.47 & 0.56 & 0.65 \\
\hline 0.37 & 0.46 & 4 & 1.25 & 0.96 & 0.65 & $\underline{0.92}$ & 0.56 & 0.37 & 0.96 \\
\hline 0.98 & 0.35 & 0.38 & 0.29 & 0.87 & 0.96 & 0.86 & 0.69 & $\underline{0.98}$ & 0.87 \\
\hline 0.47 & 0.65 & 0.56 & 0.38 & 0.57 & 0.87 & 0.13 & 0.38 & 0.47 & 0.57 \\
\hline 0.34 & 0.96 & 0.68 & 0.65 & 0.68 & 0.57 & 0.34 & 3.3 & 0.34 & 0.68 \\
\hline 0.53 & 0.87 & 0.38 & 5.69 & 0.28 & 0.38 & 0.59 & 1.56 & $\mathbf{2 . 8 5}$ & 4 \\
\hline 0.38 & $\underline{\mathbf{0 . 5 7}}$ & 0.37 & $\mathbf{1 . 3 5}$ & 4.5 & $\underline{\mathbf{5 . 0 0}}$ & 0.47 & 4 & 0.35 & 0.14 \\
\hline 0.58 & 0.68 & $\underline{0.39}$ & 7 & 4 & 0.28 & 0.56 & 0.36 & 0.98 & 0.28 \\
\hline 0.34 & 0.28 & 0.8 & 2 & 2.5 & 0.27 & 0.69 & 0.26 & $\underline{\mathbf{0 . 9 7}}$ & 0.26 \\
\hline
\end{tabular}

Figure 2: Ranked Sets (3) for initial sample.

\begin{tabular}{|r|c|c|c|c|c|c|c|c|c|}
\hline 0.32 & $\mathbf{0 . 8}$ & $\mathbf{1 . 1}$ & $\mathbf{2 . 3}$ & $\mathbf{5}$ & $\mathbf{0 . 4 6}$ & 0.13 & 0.59 & 0.32 & 0.35 \\
\hline 0.56 & $\mathbf{0 . 6}$ & $\mathbf{5}$ & $\mathbf{2 . 9 8}$ & $\mathbf{4 . 9}$ & $\mathbf{0 . 3 5}$ & 0.14 & 0.47 & 0.56 & 0.65 \\
\hline 0.37 & $\mathbf{0 . 4 6}$ & $\mathbf{4}$ & $\mathbf{1 . 2 5}$ & $\mathbf{0 . 9 6}$ & 0.65 & $\mathbf{0 . 9 2}$ & 0.56 & 0.37 & 0.96 \\
\hline 0.98 & 0.35 & $\mathbf{0 . 3 8}$ & $\mathbf{0 . 2 9}$ & 0.87 & 0.96 & 0.86 & 0.69 & 0.98 & 0.87 \\
\hline 0.47 & 0.65 & 0.56 & 0.38 & 0.57 & 0.87 & 0.13 & 0.38 & 0.47 & 0.57 \\
\hline 0.34 & 0.96 & 0.68 & $\mathbf{0 . 6 5}$ & 0.68 & 0.57 & 0.34 & 3.3 & 0.34 & 0.68 \\
\hline 0.53 & 0.87 & $\mathbf{0 . 3 8}$ & $\mathbf{5 . 6 9}$ & $\mathbf{0 . 2 8}$ & $\mathbf{0 . 3 8}$ & 0.59 & 1.56 & 2.85 & 4 \\
\hline 0.38 & 0.57 & $\mathbf{0 . 3 7}$ & $\mathbf{1 . 3 5}$ & $\mathbf{4 . 5}$ & $\mathbf{5 . 0 0}$ & $\mathbf{0 . 4 7}$ & 4.00 & 0.35 & 0.14 \\
\hline 0.58 & 0.68 & $\mathbf{0 . 3 9}$ & $\mathbf{7}$ & $\mathbf{4}$ & $\mathbf{0 . 2 8}$ & 0.56 & 0.36 & 0.98 & 0.28 \\
\hline 0.34 & 0.28 & $\mathbf{0 . 8}$ & $\mathbf{2}$ & $\mathbf{2 . 5}$ & $\mathbf{0 . 2 7}$ & 0.69 & 0.26 & 0.97 & 0.26 \\
\hline
\end{tabular}

Figure 3: Final Sample (shown in bold red-bold digits).

The population may be partitioned into $\mathrm{K}$ sets of units, termed as networks, such that selection in the initial sample of any unit in a network will result in inclusion in the final sample of all units in that network. Actually the network $A_{i}$ for unit $i$ is defined to be the cluster generated by unit $i$ but with the edge units removed. If unit $i$ is the only unit in a cluster satisfying $C$, then $A_{i}$ consists of just unit $i$ and forms a network of size one. Any unit that does not satisfy $\mathrm{C}$ to be a network of size one, as its selection does not lead to the inclusion of any other 
units. This means that all clusters of size one are also networks of size one. Here we should note that any cluster consisting of more than one unit can be split into a network and further networks of size one, as each edge units are the networks of size one. It is also clear that all the $\mathrm{K}$ networks are disjoint.

Let the final reduced sample is denoted by the unordered set $s=\left\{s_{1}, s_{2}\right\}$, where $s_{1}$ is the set of $\mathrm{n}$ unordered labels from the initial sample (which are distinct, as sampling is without replacement in the first phase), and $s_{2}$ is the set of distinct unordered labels from the remainder of the sample s. The sampling design is a function $p(\mathbf{s} \mid \mathbf{y})$ assigning a probability to every possible sample s. In designs such as those described in this paper, these selection probabilities depend on the population y-values. Let $m_{i}$ denote the number of units in $A_{i}$, and let $a_{i}$, denote the total number of units in networks of which unit $i$ is an edge unit. If unit $i$ satisfy $C$, then $a_{i}=0$, while if unit $i$ does not satisfies $C$, then $m_{i}=1$. The probability that the unit $\mathrm{i}$ is included in the sample $s_{1}$ is given by,

$$
\mathrm{p}_{i}=\frac{\mathrm{m}_{\mathrm{i}}+\mathrm{a}_{\mathrm{i}}}{\mathrm{N}}
$$

The probability that unit $\mathrm{i}$ is included in the sample $\mathrm{s}$ is

$$
\pi_{i}=1-\left[\frac{\left(\begin{array}{c}
N-m_{i}-a_{i} \\
n
\end{array}\right)}{\left(\begin{array}{l}
N \\
n
\end{array}\right)}\right] .
$$

\section{Estimators of the population mean}

Generally with the adaptive cluster sampling designs, standard estimators of the population mean and total are not unbiased. However, the classical estimators such as the sample mean $\bar{Y}$ based on simple random sampling and $\overline{\bar{Y}}$ based on the clusters with selection probabilities proportional to cluster size are unbiased under the non-adaptive designs. In this Section some estimators are given that are unbiased with the adaptive cluster sampling design discussed in this paper. These unbiased estimators do not depend on any assumptions about the population.

Let $\mathrm{S}$ denote the set of all possible samples. The expected value of an estimator $\mathrm{t}$ is defined in the design sense and is defined as,

$$
E(t)=\sum_{s \in S} t_{s} p(s \mid \mathrm{y})
$$


where $t_{s}$ is the value of the estimate for the sample $s$.

\subsection{The initial sample mean}

If the final sample of the proposed design is selected in the first phase only, the estimator of population mean $\mu$ is unbiased as ranked set sample mean is always unbiased estimator of $\mu$ for finite population. This estimator ignores all units adaptively added to the sample.

Let $y_{(i: n)}$ is the measured $y$-value of the $i^{\text {th }}$ smallest unit in the $i^{\text {th }}$ set, an unbiased estimator of $\mu$ based on the initial sample is

$$
t_{1}=\frac{1}{n} \sum_{i=1}^{n} y_{(i: n)}
$$

As each $y_{(i: n)}$ is independent and identical with mean $\mu_{(i: n)}$ (say), the variance of $t_{1}$ is given by

$$
\operatorname{Var}\left(t_{1}\right)=\frac{1}{n^{2}} \sum_{i=1}^{n} E\left(y_{(i: n)}-\mu_{(i: n)}\right)^{2}=\frac{\sigma^{2}}{n}-\frac{\sum_{i=1}^{n}\left(\mu_{(i: n)}-\mu\right)^{2}}{n^{2}},
$$

where $\sigma^{2}$ is the population variance. Generally $\mu_{(i: n)}, i=1,2, \ldots . . n$ are unknown and can be estimated by the average of the $i^{\text {th }}$ smallest ranked unit of each set.

\subsection{An estimator based on initial intersection probabilities}

If we know the inclusion probability $\pi_{i}$ that unit $\mathrm{i}$ is included in the sample $\mathrm{s}$ for all the sampled units, we can use the Horvitz-Thompson estimator, given by

$$
\hat{\mu}_{H T}=\frac{1}{N} \sum_{i \in s} \frac{y_{i}}{\pi_{i}}=\frac{1}{N} \sum_{i=1}^{N} \frac{y_{i} I_{i}}{\pi_{i}}
$$

where $I_{i}$ is the indicator variable which takes the value 1 when unit $\mathrm{i}$ is included in the sample and 0 otherwise. Unfortunately, although $m_{i}$ is known in (2.1) for all the units in s, but some of the $a_{i}$ 's are unknown. For example, if unit $i$ is an edge unit for some clusters in the sample, then all the clusters in which it belongs to, would not generally be sampled, so that $a_{i}$ will be unknown for those clusters. To 
get around this problem Thompson (1990) dropped $a_{i}$ from $\pi_{i}$ and considered the partial inclusion probability

$$
\pi_{i}^{\prime}=1-\left[\frac{\left(\begin{array}{c}
N-m_{i} \\
n
\end{array}\right)}{\left(\begin{array}{l}
N \\
n
\end{array}\right)}\right]
$$

Thus observations that do not satisfy the condition $\mathrm{C}$ are ignored if they are not included in $s_{1}$. He used the sample of $\mathrm{n}$ networks (some of which may be same), rather than the $n$ clusters, for estimating $\mu$. The probability $\pi_{i}^{\prime}$ can then be interpreted as the probability that the initial sample $s_{1}$ intersects $A_{i}$, the network for unit i.

The unbiased estimator $t_{2}$ based on the initial intersection probabilities takes the form

$$
t_{2}=\frac{1}{N} \sum_{i=1}^{N} \frac{y_{i} I_{i}^{\prime}}{\pi_{i}^{\prime}}
$$

where $I_{i}^{\prime}$ takes the value 1 (which probability $\pi_{i}^{\prime}$ ) if $s_{1}$ intersects $A_{i}$, and 0 otherwise.

It is more convenient to write the summation part of the estimator $t_{2}$ in (3.6) in terms of the distinct networks, as the intersection probability $\pi_{i}^{\prime}$ is same $\left(=\alpha_{k}\right.$, say) for each unit $\mathrm{i}$ in the $k^{\text {th }}$ network. Hence

$$
t_{2}=\frac{1}{N} \sum_{k=1}^{K} \frac{y_{k}^{*} J_{k}}{\alpha_{k}}=\frac{1}{N} \sum_{k=1}^{K^{\prime}} \frac{y_{k}^{*}}{\alpha_{k}},
$$

where $y_{k}^{*}$ is the sum of the $\mathrm{y}$-values for the network $\mathrm{k}, \mathrm{K}$ is the total number of distinct networks in the population, $K^{\prime}$ is the number of distinct networks in the sample s, and $J_{k}$ takes a value of 1 (with probability $\alpha_{k}$ ) if the initial sample intersects the network $\mathrm{k}$, and 0 otherwise. If there are $x_{k}$ units in the network $\mathrm{k}$, then 


$$
\alpha_{k}=1-\left[\left(\begin{array}{c}
N-x_{k} \\
n
\end{array}\right) /\left(\begin{array}{l}
N \\
n
\end{array}\right)\right]
$$

Also letting $p_{j k}=\mathrm{P}\left(j^{\text {th }}\right.$ and $k^{\text {th }}$ network not intersected $)$, then

$$
p_{j k}=P\left(J_{j} \neq 1 \cap J_{k} \neq 1\right)=\frac{\left(\begin{array}{c}
N-x_{j}-x_{k} \\
n
\end{array}\right)}{\left(\begin{array}{l}
N \\
n
\end{array}\right)} .
$$

Therefore the probability that the networks $\mathrm{j}$ and $\mathrm{k}$ are both intersected is

$$
\begin{aligned}
& \alpha_{j k}=P\left(J_{j}=1 \cup J_{k}=1\right) \\
& =P\left(J_{j}=1\right)+P\left(J_{k}=1\right)-P\left(J_{j}=1 \cap J_{k}=1\right) \\
& =\alpha_{j}+\alpha_{k}-\left(1-p_{j k}\right)
\end{aligned}
$$

or

$$
\alpha_{j k}=1-\frac{\left[\left(\begin{array}{c}
N-x_{j} \\
n
\end{array}\right)+\left(\begin{array}{c}
N-x_{k} \\
n
\end{array}\right)-\left(\begin{array}{c}
N-x_{j}-x_{k} \\
n
\end{array}\right)\right]}{\left(\begin{array}{l}
N \\
n
\end{array}\right)} .
$$

With the convention that $\alpha_{j j}=\alpha_{j}$, the variance of $t_{2}$ is

$$
\operatorname{Var}\left(t_{2}\right)=\frac{1}{N^{2}}\left[\sum_{j=1}^{K} \sum_{k=1}^{K} y_{j}^{*} y_{k}^{*}\left(\frac{\alpha_{j k}-\alpha_{j} \alpha_{k}}{\alpha_{j} \alpha_{k}}\right)\right] .
$$

An unbiased estimator of the variance of $t_{2}$ is

$$
\begin{aligned}
\hat{\operatorname{Var}}\left(t_{2}\right) & =\frac{1}{N^{2}}\left[\sum_{j=1}^{K} \sum_{k=1}^{K} y_{j}^{*} y_{k}^{*}\left(\frac{\alpha_{j k}-\alpha_{j} \alpha_{k}}{\alpha_{j k} \alpha_{j} \alpha_{k}}\right) J_{j} J_{k}\right] \\
& =\frac{1}{N^{2}}\left[\sum_{j=1}^{K^{\prime}} \sum_{k=1}^{K^{\prime}} y_{j}^{*} y_{k}^{*}\left(\frac{\alpha_{j k}-\alpha_{j} \alpha_{k}}{\alpha_{j k} \alpha_{j} \alpha_{k}}\right)\right],
\end{aligned}
$$

provided that none of the joint probabilities $\alpha_{j k}$ is zero.

Just as the Horvitz-Thompson estimator has small variance when the y-values are approximately proportional to the inclusion probabilities, the estimator $t_{2}$ 
should have low variance when the network totals $y_{k}^{*}$ 's are proportional to the intersection probability $\alpha_{k}$.

\subsection{Improvement of the estimators using the Rao-Blackwell method}

The estimators $t_{1}$ and $t_{2}$ of $\mu$ are although unbiased but are not the function of the minimal sufficient statistic, and so each may be improved by the Rao-Blackwell theorem by taking conditional expectation, given the minimal sufficient statistic. For finite population Basu (1969) suggested that the minimal sufficient statistic D is the unordered set of distinct, labelled observations, that is,

$$
D=\left\{\left(k, y_{k}\right): k \in s\right\}
$$

Starting with any one of the unbiased estimators $t_{R B}=E(t \mid D)$. Let $n^{\prime}$ denote the number of distinct units in the final adaptive sample s. If the initial sample $s_{1}$ is selected without replacement, define $G=\left(\begin{array}{l}n^{\prime} \\ n\end{array}\right)$, the number of possible combinations of $\mathrm{n}$ distinct units from the $n^{\prime}$ in the sample. Suppose that these combinations are indexed in an arbitrary way by the label $\mathrm{g}(\mathrm{g}=1,2 \ldots \mathrm{G})$. Let $t_{g}$ denote the value of $\mathrm{t}$ when $s_{1}$ consists of combination $\mathrm{g}$ and let $\hat{\operatorname{Var}}_{g}(t)$ denote the value of the unbiased estimator $\hat{\operatorname{Var}}(t)$, when computed using the $g^{\text {th }}$ combination.

An initial sample that gives rise through the design to the given value $\mathrm{D}$ of the minimal sufficient statistic is called compatible with D. Let the $g^{\text {th }}$ indicator variable $\left(I_{g}\right)$ takes the value 1 if the $g^{\text {th }}$ combination could give rise to D (i.e., is compatible with D), and 0 otherwise. The number of compatible combinations is then

$$
\xi=\sum_{g=1}^{G} I_{g}
$$

Now the estimator $t$ may be improved using Rao-Blackwell theorem and is the average of the values of $t$ obtained over all those initial samples that are compatible with D. This improved estimator $t_{R B}$ is

$$
t_{R B}=E(t \mid D)=\frac{1}{\xi} \sum_{g=1}^{\xi} t_{g}
$$

or 


$$
t_{R B}=\frac{1}{\xi} \sum_{g=1}^{G} t_{g} I_{g}
$$

and its variance is given by

$$
\operatorname{Var}\left(t_{R B}\right)=\operatorname{Var}(t)-E[\operatorname{Var}(t \mid D)] \text {. }
$$

An unbiased estimator of the variance of $t_{R B}$ due to Thompson (1990) is given by

$$
\hat{\operatorname{Var}}\left(t_{R B}\right)=\frac{1}{\xi} \sum_{g=1}^{G}\left[\hat{\operatorname{Var}}\left(t_{g}\right)-\left(t_{g}-t_{R B}\right)^{2}\right] I_{g}
$$

\section{Example}

To demonstrate the utility of the proposed procedure and its superiority over the existing procedures, we use a small artificial population of five units with y-values $\mathbf{y}=(2,500,3,15,4)^{\prime}$. The neighbourhood of each unit includes its immediately adjacent units (of which there are either one or two units). The condition $\mathrm{C}$ is defined by $\mathrm{C}=\{y: y \geq 5\}$ and the initial sample size $\mathrm{n}=2$.

With the proposed design in which the initial sample is selected by ranked set sampling, there are $\left(\begin{array}{l}5 \\ 2\end{array}\right)\left(\begin{array}{l}3 \\ 2\end{array}\right)=30$ possible combinations of units; each combination has two sets, each of size 2. All 30 possible initial samples from these combinations are given in the first column of Table 1 . The final sample is given in the second column of the Table 1 . In this population the $1^{\text {st }}, 2^{\text {nd }}$ and $3^{\text {rd }}$ units, with $y$-values 2, 500, and 3, form a cluster consisting of 3 networks each of size 1 .

In the first row of the Table 1 , the $1^{\text {st }}$ and $2^{\text {nd }}$ units of the population, with yvalues 2 and 500, are selected initially. Since $500 \geq 5$, the single neighbour of the $2^{\text {nd }}$ unit, having $y$-value 3 , is then added to the sample. The other neighbours of $2^{\text {nd }}$ unit, having y-value 2 is selected as a member of initial sample, hence is not selected again. Ignoring the edge units, the results for the estimators of first sample (i.e. of the first row of Table 1) are given below:

The initial sample mean $t_{1}=251$.

The values $\alpha_{1}$ and $\alpha_{2}$ are $\alpha_{1}=0.4$ and $\alpha_{2}=0.7$, leading to $t_{2}=251$.

The classical estimators are $\bar{y}=168.3$ and $\bar{y}=168.3$.

The values of the Rao-Blackwell version of any of the estimators for each sample are obtained by averaging the value of the corresponding estimator over those samples that are compatible with D. For the first sample of this example

$$
t_{1 R B}=251.25 \text { and } t_{2 R B}=251.25 \text {. }
$$


Table 1: Observations under the proposed procedure.

\begin{tabular}{|c|c|c|c|c|c|c|c|c|}
\hline S.No & $s_{1}$ & $S_{2}$ & $t_{1}$ & $t_{2}$ & $t_{1 R B}$ & $t_{2 R B}$ & $\bar{y}$ & $\bar{y}$ \\
\hline 1 & $(2,500)$ & $(2,500,3)$ & 251.0 & 251.0 & 251.3 & 251.3 & 168.3 & 168.3 \\
\hline 2 & $(2,15)$ & $(2,15,3,4)$ & 8.5 & 8.5 & 8.5 & 8.5 & 6.0 & 4.7 \\
\hline 3 & $(2,500)$ & $(2,500,3)$ & 251.0 & 251.0 & 251.3 & 251.3 & 168.3 & 168.3 \\
\hline 4 & $(2,500)$ & $(2,500,3)$ & 251.0 & 251.0 & 251.3 & 251.3 & 168.3 & 168.3 \\
\hline 5 & $(2,4)$ & $(2,4)$ & 3.0 & 3.0 & 3.0 & 3.0 & 3.0 & 3.0 \\
\hline 6 & $(2,500)$ & $(2,500,3)$ & 251.0 & 251.0 & 251.3 & 251.3 & 168.3 & 168.3 \\
\hline 7 & $(2,15)$ & $(2,15,3,4)$ & 8.5 & 8.5 & 8.5 & 8.5 & 6.0 & 4.7 \\
\hline 8 & $(2,4)$ & $(2,4)$ & 3.0 & 3.0 & 3.0 & 3.0 & 3.0 & 3.0 \\
\hline 9 & $(2,15)$ & $(2,15,3,4)$ & 8.5 & 8.5 & 8.5 & 8.5 & 6.0 & 4.7 \\
\hline 10 & $(2,15)$ & $(2,15,3,4)$ & 8.5 & 8.5 & 8.5 & 8.5 & 6.0 & 4.7 \\
\hline 11 & $(2,500)$ & $(2,500,3)$ & 251.0 & 251.0 & 251.3 & 251.3 & 168.3 & 168.3 \\
\hline 12 & $(2,500)$ & $(2,500,3)$ & 251.0 & 251.0 & 251.3 & 251.3 & 168.3 & 168.3 \\
\hline 13 & $(3,500)$ & $(3,500,2)$ & 251.5 & 251.5 & 251.3 & 251.3 & 168.3 & 168.3 \\
\hline 14 & $(3,15)$ & $(3,15,4)$ & 9.0 & 9.0 & 9.3 & 9.3 & 22.0 & 7.3 \\
\hline 15 & $(3,500)$ & $(3,500,2)$ & 251.5 & 251.5 & 251.3 & 251.3 & 168.3 & 168.3 \\
\hline 16 & $(15,3)$ & $(15,3,4)$ & 9.0 & 9.0 & 9.3 & 9.3 & 22.0 & 7.3 \\
\hline 17 & $(4,3)$ & $(4,3)$ & 3.5 & 3.5 & 3.5 & 3.5 & 3.5 & 3.5 \\
\hline 18 & $(4,3)$ & $(4,3)$ & 3.5 & 3.5 & 3.5 & 3.5 & 3.5 & 3.5 \\
\hline 19 & $(3,15)$ & $(3,15,4)$ & 9.0 & 9.0 & 9.3 & 9.3 & 22.0 & 7.3 \\
\hline 20 & $(3,15)$ & $(3,15,4)$ & 9.0 & 9.0 & 9.3 & 9.3 & 22.0 & 7.3 \\
\hline 21 & $(4,15)$ & $(4,15,3)$ & 9.5 & 9.5 & 9.3 & 9.3 & 168.3 & 7.3 \\
\hline 22 & $(3,500)$ & $(3,500,2)$ & 251.5 & 251.5 & 251.3 & 251.3 & 168.3 & 168.3 \\
\hline 23 & $(3,500)$ & $(3,500,2)$ & 251.5 & 251.5 & 251.3 & 251.3 & 168.3 & 168.3 \\
\hline 24 & $(4,500)$ & $(4,500,2,3)$ & 252.0 & 252.0 & 252.0 & 252.0 & 127.3 & 86.2 \\
\hline 25 & $(3,4)$ & $(3,4)$ & 3.5 & 3.5 & 3.5 & 3.5 & 3.5 & 3.5 \\
\hline 26 & $(3,4)$ & $(3,4)$ & 3.5 & 3.5 & 3.5 & 3.5 & 3.5 & 3.5 \\
\hline 27 & $(15,4)$ & $(15,4,3)$ & 9.5 & 9.5 & 9.3 & 9.3 & 168.3 & 7.3 \\
\hline 28 & $(4,15)$ & $(4,15,3)$ & 9.5 & 9.5 & 9.3 & 9.3 & 168.3 & 7.3 \\
\hline 29 & $(4,500)$ & $(4,500,2,3)$ & 252.0 & 252.0 & 252.0 & 252.0 & 127.3 & 86.2 \\
\hline \multirow[t]{3}{*}{30} & $(15,4)$ & $(15,4,3)$ & 9.5 & 9.5 & 9.3 & 9.3 & 168.3 & 7.3 \\
\hline & MEAN & & 104.8 & 104.8 & 104.8 & 104.8 & 91.4 & 65.1 \\
\hline & BIAS & & 0.0 & 0.0 & 0.0 & 0.0 & -13.4 & -39.7 \\
\hline
\end{tabular}

The population mean is 104.8 , and the population variance is 48834.7 . From Table 1, it is clear that the estimators $t_{1}, t_{2}, t_{1 R B}$ and $t_{2 R B}$ are unbiased, whereas the estimators $\bar{y}$ and $\bar{y}$, used with adaptive design are biased. 
With the adaptive design in which the initial sample is selected by SRS without replacement, there are $\left(\begin{array}{l}5 \\ 2\end{array}\right)=10$ possible samples, each having probability 1/10. The final sample and the values of each estimator with mean and variance are listed in Table 2.

Table 2: Observations under Thompson's adaptive design.

\begin{tabular}{rcrrrrr} 
S.No. & \multicolumn{1}{c}{$S_{2}$} & \multicolumn{1}{c}{$t_{1}$} & \multicolumn{1}{c}{$t_{2}$} & \multicolumn{1}{c}{$t_{1 R B}$} & \multicolumn{1}{c}{$t_{2 R B}$} & \multicolumn{1}{c}{$y$} \\
1 & $(2,500,3)$ & 251.00 & 143.86 & 251.25 & 144.11 & 168.33 \\
2 & $(2,3)$ & 2.50 & 2.50 & 127.00 & 73.43 & 2.5 \\
3 & $(2,15,4)$ & 8.50 & 5.29 & 4.25 & 2.64 & 7 \\
4 & $(2,4)$ & 3.00 & 3.00 & 127.50 & 73.93 & 3 \\
5 & $(500,3,2)$ & 251.50 & 144.36 & 251.25 & 144.11 & 168.33 \\
6 & $(500,15,2,3,4)$ & 257.50 & 147.14 & 257.50 & 147.14 & 104.8 \\
7 & $(500,4,2)$ & 252.00 & 144.86 & 254.75 & 146.00 & 168.67 \\
8 & $(3,15,4)$ & 9.00 & 5.79 & 8.75 & 6.04 & 7.33 \\
9 & $(3,4)$ & 3.50 & 3.50 & 7.00 & 5.19 & 3.5 \\
10 & $(15,4,3)$ & 8.50 & 6.29 & 8.75 & 6.04 & 7.33 \\
& MEAN & 104.70 & 60.66 & 129.80 & 74.86 & 64.08
\end{tabular}

From Table 1 and Table 2, it may be concluded that for this small artificial example, the proposed procedure gives more average yield for almost all the estimators in comparison to that given by the procedure of Thompson (1990).

\section{Conclusion}

When the measurements of units are very costly and time consuming and there is heterogeneity between the units of the population, the simple random sampling become useless. In such situations, RSS is a cost-effective and precise method of sample selection. In this discussion, we have used the technique of RSS to select the initial sample under adaptive sampling. The proposed design is more efficient than adaptive cluster sampling based on simple random sampling for estimating rare and endangered population, under the assumption that ranking of sampling units are easier than actual measurements. It also contains the information about all order statistics. Relative precision of RSS compared with SRS is an increasing function of set size $(\mathrm{k})$. It shows that the efficiency of proposed design increases as $\mathrm{k}$ in the first phase of the design increases. We have demonstrated theoretically as well as with the help of an artificial example that the proposed procedure is more advantageous in comparison to existing procedures of adaptive sampling in the 
sense that it provides unbiased estimators as well as it give equal importance to all the rank orders and as such it is more informative. The proposed design also establishes a bridge between the ranked set sampling and adaptive cluster sampling and uses the advantage of both the schemes. However, data availability in the form of ranked clusters has to be determined in advance. As there are only few sampling procedures in the area of rare species estimation as well as when the measurement of units are difficult and costly in comparison to the ranking of units by inexpensive techniques including visual inspection, the doors are open in future to develop the strategy for extracting the benefits of the two schemes. The proposed procedure has also particular relevance to assess the effects of human induced activities in the flora, fauna etc., that increase species rarity.

\section{Acknowledgement}

The authors are grateful to the Editor and an anonymous referee for their constructive comments and suggestions, which led to considerable improvement in presentation of this manuscript. We are also thankful to Ministry of Statistics and Program Implementation, Government of India for providing the fund to present the paper in the International Conference on Applied Statstics-2010 at Ribno, Slovenia.

\section{References}

[1] Barnett, V. and Moore, K. (1997): Best linear unbiased estimates in ranked set sampling with particular reference to imperfect ordering. Journal of Applied Statistics, 24, 697-710.

[2] Basu, D. (1969): Role of the sufficiency and likelihood principle in sample survey theory. Sankhya, 31(A), 441-454.

[3] Blanke, D. (2006): Adaptive sampling schemes for density estimation. Journal of Statistical Planning and Inference, 136, 2898-2917.

[4] Chaudhuri, A., Bose, M., and Ghosh, J.K. (2004): An application of adaptive sampling to estimate highly localized population segments. Journal of Statistical Planning and Inference, 121, 175-189.

[5] Chen, Z., Bai, Z.D., and Sinha, B.K. (2004): Ranked Set Sampling: Theory and Applications. New York: Springer-Verlag.

[6] Cobby, J.M., Ridout, M.S., Bassett, P.J., and Large, R.V. (1985): An investigation into the use of ranked set sampling on grass and grass-clover swards. Grass and Forage Science, 40, 257-263.

[7] Halls, L.K. and Dell, T.R. (1966): Trials of ranked set sampling for forage yields. Forest Science, 12, 22-26. 
[8] $\mathrm{Hu}$, J. and Su, Z. (2007): Adaptive resampling algorithms for estimating bootstrap distributions. Journal of Statistical Planning and Inference, article in press.

[9] Kaur, A., Patil, G.P., Sinha, A.K., and Taillie, C. (1995): Ranked set sampling, an annotated bibliography. Environmental and Ecological Statistics, 2, 25-54.

[10] Martin, W.L., Sharik, T.L., Oderwald, R.G., and Smith, D.W. (1980): Evaluation of Ranked Set Sampling for Estimating Shrub Phytomass in Appalachian Oak Forests. Blacksburg, Virginia: School of Forestry and Wildlife Resources, Virginia Polytechnic Institute and State University, FWS, 4-80.

[11] McIntyre, G.A. (1952): A method for unbiased selective sampling using ranked sets. Australian Journal of Agricultural Research, 3, 385-390.

[12] McIntyre, G.A. (1978): Statistical aspects of vegetation sampling. In Mannetje, L.T. (Ed.): Measurement of Grassland Vegetation and Animal Production Bulletin, 52, Hurley, Berkshire: Commonwealth Bureau of Pastures and Field Crops. 8-21.

[13] Muttlak, H.A. and McDonald, L.L. (1992): Ranked set sampling and the line intercept method: A more efficient procedure. Biometrical Journal, 34, 329346.

[14] Nahhas, R.W., Wolfe, D.A., and Chen, H. (2002): Ranked set sampling: Cost and optimal set size. Biometrics, 58, 964-971.

[15] Patil, G.P., Sinha, A.K., and Taillie, C. (1994): Ranked set sampling. In Patil, G.P. and Rao, C.R. (Eds.): Handbook of Statistics, New York: Elsevier Science Publishers, 167-199.

[16] Roesch, F.A., Jr. (1993): Adaptive cluster sampling for forest inventories. Forest Science, 39, 655-669.

[17] Salehi, M.M. and Seber, G.A.F. (1997): Two stage adaptive cluster sampling. Biometrics, 53, 959-970.

[18] Salehi, M.M. and Seber, G.A.F. (2004): A general inverse sampling scheme and its application to adaptive cluster sampling. Australian and New Zealand Journal of Statistics, 46, 483-494.

[19] Stokes, S.L. and Sager, T.W. (1988): Characterization of a ranked set sample with application to estimating distribution functions. Journal of the American Statistical Association, 83, 374-381.

[20] Thompson, S.K. (1990): Adaptive cluster sampling. Journal of the American Statistical Association, 85, 1050-1059.

[21] Thompson, S.K. (1991a): Stratified adaptive cluster sampling. Biometrika, 78, 389-397.

[22] Thompson, S.K. (1991b): Adaptive cluster sampling: Designs with primary and secondary units. Biometrics, 47, 1103-1115. 
[23] Thompson, S.K. (1996): Adaptive cluster sampling based on order statistics. Environmetrics, 7, 123-133.

[24] Thompson, S.K. and Seber, G.A.F. (1996): Adaptive Sampling, New York: Wiley. 